\title{
Hydroaktives Polyurethanpflaster zur Behandlung hypertropher Narben und Keloide
}

\author{
H. Mensing ${ }^{1}$ \\ J. Ortega² \\ O. Hoch ${ }^{2}$ \\ H. U.von Sobbe ${ }^{2}$ \\ C. H. Mensing ${ }^{2}$
}

\author{
Hydroactive polyurethan dressing for treatment of hypertrophic scars \\ and keloids
}

\section{Zusammenfassung}

Zur Beurteilung der Wirksamkeit eines hydroactiven Polyurethanpflasters bei hypertrophen Narben und Keloiden wurde eine offene Studie an 46 Patienten durchgeführt. Eine Verbesserung der klinischen Zielparameter ließ sich bei einer Behandlungszeit von 8-12 Wochen bei nahezu allen Patienten feststellen, wobei die Handhabung problemlos und einfach, die Verträglichkeit überwiegend sehr gut war. Das hier geprüfte Polyurethanpflaster stellt somit eine zusätzliche Alternative in der immer noch schwierigen Behandlung keloidaler Narben dar.

\section{Abstract}

For evaluation of the effectivness of a hydroactive polyurethan plaster in the treatment of keloid scars an open study was conducted. 46 patients were included. An improvement of the clinical parameters could be detected in nearly all participants during the study time of 8-12 weeks. The handling of the plaster was easy, the tolerability mostly good. Therefore this polyurethan dressing seems to be a further alternative in the still difficult therapy of keloid scars.

\section{Einleitung}

Hypertrophe Narben und Keloide sind Regulationsstörungen der Narbenbildung mit der Folge vermehrter Kollagenbildung, die sich in Form wulstiger, hautfarbener oder auch livid-rötlicher Papeln und Tumoren über die Hautoberfläche erheben. Die letztendliche Ursache dieser Regulationsstörung ist unbekannt, lokalisatorische und genetische Faktoren (familiäre Häufung, Bevorzugung Hauttyp 3-6) scheinen aber wichtige Grundvoraussetzungen zu sein[3]. Eine Unterscheidung hypertropher Narben und Keloide erfolgt vor allen Dingen klinisch, da Keloide meist im Sinne eines benignen Tumors deutlich über die eigentlichen Narbenkonturen hinauswachsen und eher selten zu spontanen Involutionen neigen. Größe, Farbe, Konsistenz und Sensibilität (Pruritus) sind aber von graduellem Unterschied, auch histologisch ergeben sich viele Übereinstimmungen in der Gesamtstruktur, wobei Keloide zellärmer und gröbere homogenisierte kollagene Fasern aufweisen, während hypertrophe Narben meist einen höheren Fibroblastenreichtum aufweisen [5,20].

Außer einem mehr oder minder ausgeprägten Juckreiz und im Extremfall dermatogenen Kontrakturen, falls Hautpartien über Gelenkflächen von dieser Narbenwucherung betroffen sind, sind keloide Narben vor allem kosmetisch störende und beeinträchtigende Hautveränderungen, deren Behandlung aber trotz ihres relativ häufigen Auftretens nach wie vor ein nicht unerhebliches medizinisches Problem darstellen.

Konservative Maßnahmen mit Auftragen diverser Externa und Druckverbände [7], Unterspritzung intraläsional mit Kortikoidkristallsuspension [11], 5 Fluor-Uracil [8] oder neuerdings auch Pricken mit Bleomycin [6] sowie Auflagen mit Silikonpflastern [10] haben zwar partielle Erfolge zu verzeichnen, sind aber in der Regel weit entfernt von kosmetisch befriedigenden oder gar 
kurativen Effekten. Das Gleiche gilt für aggressivere Verfahren wie Reoperation, Kryochirurgie [17], Laser-Anwendung [12] oder die heute kaum noch geübte niedrig dosierte Röntgenbestrahlung (3 - 4-mal 400 R). Das grundsätzliche Problem aller invasiven Methoden liegt in der Induktion neuer Reparationsvorgänge mit neuerlicher, unkontrollierter Kollagenneogenese, die z.T. zu noch größeren Narbenbildungen als zuvor vorhanden führen können.

Mit der Entwicklung eines hydroaktiven Polyurethanpflasters (Hansaplast Narbenreduktion) besteht eine weitere Möglichkeit, Narbengewebe durch Auflage eines semiokklusiv wirkenden Pflasters konservativ zu beeinflussen.

Um die Effektivität dieses narbenreduzierenden Pflasters besser einschätzen zu können, wurde eine offene Studie mit 46 Patienten konzipiert, über deren Ergebnisse nachfolgend berichtet wird.

\section{Material und Methoden}

\section{Allgemeine Parameter}

Die Prüfung erfolgte in der Zeit von Oktober 2000 bis April 2001. Die Prüfungszeit betrug 8-12 Wochen. Das Polyurethanpflaster - Hansaplast Narbenreduktion - wurde von der betroffenen Person in der Größe der keloidalen Narben ausgeschnitten, über 24 Stunden mit oder ohne Fixiermaterial auf die Narbe aufgeklebt, nach diesem Zeitraum abgenommen und in gleicher Weise erneuert. Zeitaufwand: wenige Minuten.

Ausschlusskriterien bestanden lediglich bei bekannter Unverträglichkeitsreaktion auf das Material oder nicht ausreichende Kooperationsfähigkeit der Patienten.

46 Personen, 13 Männer und 33 Frauen, wurden in die Studie eingeschlossen, der jüngste Patient war 8 Jahre, der älteste 77 Jahre alt, im Durchschnitt ergab sich ein Alter von 42,7 $\pm 22,6$ Jahre, Median 39 Jahre. Keiner der Patienten wies anamnestisch wesentliche dermatologische Erkrankungen oder allergische Reaktionen auf.

Auf eine Unterteilung in hypertrophe Narbe und Keloide wurden bei dem letztlich unscharfen Unterscheidungskriterium verzichtet, vielmehr wurden als Einschlusskriterien Aktivitätsparameter wie Juckreiz, Rötung, Spannungsgefühl etc. (s.u.) gewählt, um die klinische Effizienz insbesondere im Hinblick auf die subjektiven Beschwerden der Patienten zu ermitteln. Im Folgenden werden daher die Narben als keloidale Narben (KN) bezeichnet.

Als Ursache der KN wurden in $72 \%$ chirurgische Eingriffe, in $4 \%$ Trauma, in den übrigen $24 \%$ keine verwertbaren Ursachen angegeben. Die Hauptlokalisationen waren Brust (26\%), Bauch (15\%), Oberarm und Schultern (je 11\%), Dekollete und Oberschenkel (9\%).

Eine Vorbehandlung war lediglich bei 3 der 46 Patienten erfolgt, allerdings ohne jeglichen Effekt, diese Behandlung waren mindestens 3 Wochen vorher beendet worden. Kontrolluntersuchungen fanden nach 4 und 8 Wochen, bei einigen Patienten nach weiteren 4 Wochen, also nach 12 Wochen, statt; eine Verlängerung der Anwendung erfolgte bei solchen Patienten, die subjektiv das Gefühl hatten, dass eine weitere Verbesserung des Narbenzustandes zu erwarten war (15 Patienten). Die übrigen 31 Patienten hingegen meinten, nach 8 Wochen keine Veränderung des Narbenbildes mehr festzustellen bzw. zu erwarten.

\section{Eigenschaften der keloidalen Narben (KN)}

Zur Kontrolle des Therapieeffektes wurden zu Beginn der Behandlung Größe und Eigenschaft der Keloide festgestellt. Die Breite der KN reichte von 0,2 - $6 \mathrm{~cm}$ (Mittelwert 1,6 cm), die Länge von $0,3-11,5 \mathrm{~cm}$ (Mittelwert: $2,2 \mathrm{~cm}$ ), die Fläche betrug $0,2-30 \mathrm{~cm}^{2}$ (Mittelwert $3,3 \mathrm{~cm}^{2}$ ). Die Eigenschaften Rötung $100 \%$, Wulstigkeit 100\%, Rauigkeit 48\%, Spannungsgefühl $52 \%$, Juckreiz $63 \%$ wurden erfasst und nach den Kriterien „nicht vorhanden“, „wenig“, „mäßig“ und „stark“ skaliert. Die Ausgangsbefunde sowie deren Beeinflussung während und nach Ende der Behandlung lassen sich aus Abb.1 ablesen. Fotodokumentation erfolgte jeweils vor Beginn und nach Ende der Therapie.

\section{Ergebnisse}

Die Ergebnisse nach 4 und 8 Wochen bzw. nach einem Zusatzbesuch nach 12 Wochen (15 Patienten) sind in Abb. 1 dargestellt. Es zeigt sich, dass die 5 Zielparameter Rötung, Wulstigkeit, Rauigkeit, Spannungsgefühl, Juckreiz bereits nach 4 Wochen deutlich positiv durch das Aufkleben des Narbenpads beeinflusst wurden, da mehr als drei Viertel der behandelten Personen bereits zu diesem Zeitpunkt Verbesserungen bemerkten. Dieser Trend setzte sich verstärkt bis zur achten Woche fort, so dass zu diesem Zeitpunkt 34 Patienten die Behandlung beendeten, da sie mit der erreichten Verbesserung zufrieden waren oder sie in den letzten zwei Wochen der Behandlung keine weitere Verbesserung mehr feststellen konnten. 15 Patienten mit einem eher etwas verzögertem Wirkeintritt hatten dagegen den Eindruck, dass auch in den letzten 2 Wochen der Anwendung noch Verbesserungen der genannten Zielparameter zu vermerken waren, so dass sie die Behandlung noch weitere 3-4 Wochen fortsetzten. Dies ließ sich insofern als richtig bestätigen, als praktisch von allen diesen 15 Personen weitere Verbesserungen der Narbeneigenschaften bewertet wurden, so dass die Gesamtbeurteilung des Narbenpads am Ende der Behandlung von 21,7\% als normalisiert im Sinne einer flachen, reizlosen Narbe und 78,3\% als verbessert angegeben wurde (Abb. 2).

Eine gesonderte Beurteilung der Wirksamkeit des Pflasters nach der Zeitdauer des Bestehens der KN - kürzer oder länger als $1 \mathrm{Jahr}$ - ergab keine wesentlichen Unterschiede in Bezug auf die Zielparameter. Diese wurden in gleicher Weise bei jüngeren und älteren $\mathrm{KN}$ verbessert.

Die Beurteilung der Verträglichkeit wurde unterschieden in die Verträglichkeit des Narbenpads und des Fixiermaterials (Tab.1). 12 Personen hatten das Fixiermaterial nicht genutzt, da die Haftfähigkeit des Narbenpads ihnen auch so hinreichend war. Das Narbenpad alleine wurde von praktisch allen Patienten problemlos vertragen, während das Fixiermaterial von 4 von 34 Patienten nicht oder nur mäßig vertragen wurde (Juckreiz, Rötung). Die Abschlussbefragung, ob die Patienten dieses Narbenpad erneut 

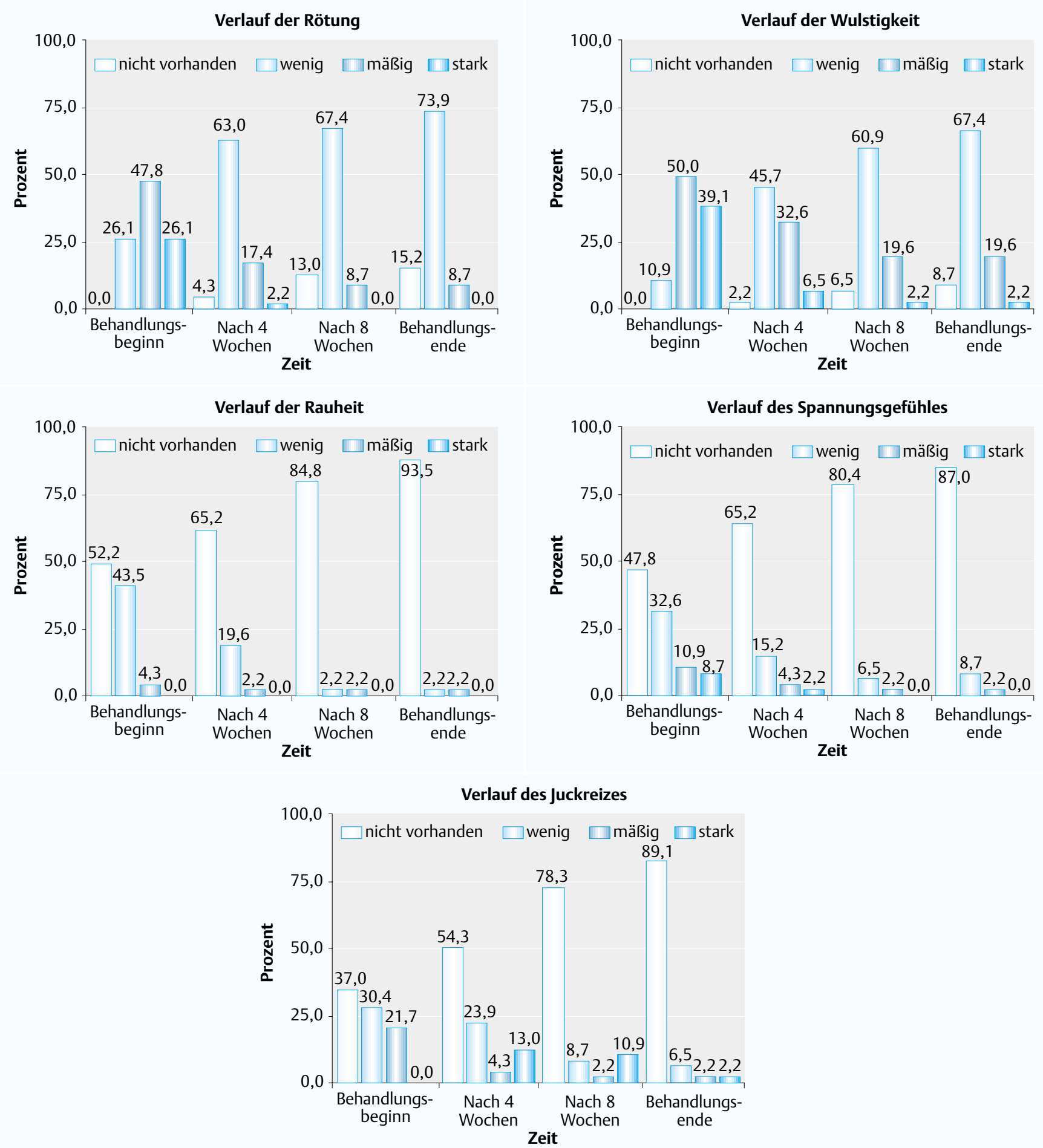

Abb. 1 Gegenüberstellung der Ergebnisse in Bezug auf die 5 untersuchten Parameter (Rötungen, Wulstigkeit, Rauigkeit, Spannungsgefühl und Juckreiz) zu verschiedenen Behandlungszeitpunkten.

anwenden würden, beantworteten 38 von 39 Patienten mit „ja“, bei 7 Patienten wurde keine Angabe registriert.

\section{Besprechung}

Die vorliegenden Untersuchungsergebnisse zur Wirksamkeit und Verträglichkeit des hydroaktiven Polyurethanpflasters zur Reduktion von keloidalen Narben wurde auf der Basis einer offe- nen Anwendungsbeobachtung gewonnen; die Zielparameter Rötung, Wulstigkeit, Rauigkeit, Spannungsgefühl und Juckreiz wurden nach subjektiven Angaben der Patienten und nach Inaugenscheinnahme des Untersuchers und Fotodokumentation beurteilt. Da es sich um eine Studie unter Praxisbedingungen handelte, die die im täglichen Gebrauch sich darstellenden Effekte registrieren sollte, wurden keine zusätzlich objektivierbaren Methoden, wie z.B. Ultraschalluntersuchung etc. des Narbengewebes durchgeführt, so dass das Studiendesign hierzu keine me- 


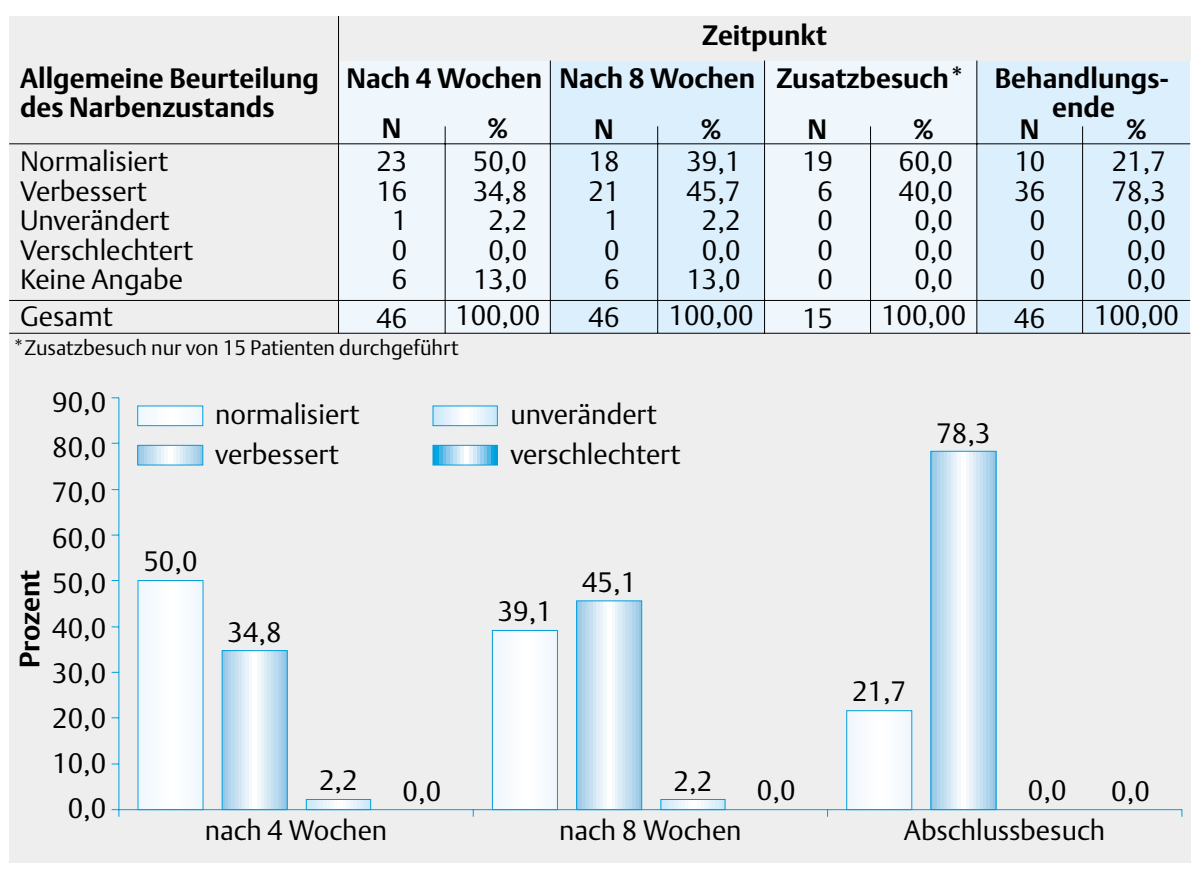

Abb. 2 Allgemeine Beurteilung des Narbenzustandes.
Tab. 1 Beurteilung der Verträglichkeit

\begin{tabular}{lrrrr}
\hline $\begin{array}{l}\text { allgemeine Beurteilung } \\
\text { der Verträglichkeit } \\
\text { des Produktes }\end{array}$ & \multicolumn{1}{c}{ Fixiermaterial } & \multicolumn{2}{l}{ Narbenpad } \\
\hline sehr gut & $\boldsymbol{n}$ & $\%$ & $\boldsymbol{n}$ & \multicolumn{1}{c}{$\%$} \\
\hline gut & 21 & 45,7 & 39 & 84,8 \\
\hline mäßig & 8 & 17,4 & 4 & 8,7 \\
\hline schlecht & 1 & 2,2 & 0 & 0,0 \\
\hline keine Angabe & 3 & 6,5 & 0 & 0,0 \\
\hline nicht benutzt & 1 & 2,2 & 3 & 6,5 \\
\hline gesamt & 12 & 26,1 & & \\
\hline
\end{tabular}

trisch-objektivierbaren Daten liefern kann. Dennoch liefert das Ergebnis dieser Untersuchung interessante und verwertbare Erkenntnisse, da gerade die häufig vorkommenden und kosmetisch stark störenden Keloide bei praktisch allen Patienten eine subjektiv deutliche Verbesserung erfahren hatten, so dass dem Narbenpad eine ausgesprochen hohe Akzeptanz und Wirksamkeit entgegengebracht und bescheinigt wurde. Ausweislich der fotografischen Dokumentation lassen sich aber auch ganz offensichtlich hervorragend objektivierbare Ergebnisse durch die Behandlung mit dem Narbenpad (Hansaplast Narben Reduktion) erzielen (Abb.3-6). Der Eintritt der Wirksamkeit erfolgte bei zwei Drittel der Patienten nach $2-3$ Wochen und führte bei einer zweimonatigen Anwendung zu einem stabilen Ergebnis, so dass die Behandlung zu diesem Zeitpunkt beendet werden konnte.

Bei ca. einem Drittel war die Wirksamkeit verzögert, so dass eine Verlängerung der Anwendung über weitere 4 Wochen zu gleich guten Ergebnissen führte. Eine Differenzierung, bei welchen Patienten oder welcher Art von Keloid mit einer solchen Verzögerung zu rechnen ist, lässt sich aus den hier vorliegenden Daten leider nicht geben, da dieses weder durch Geschlecht, Größe, Lokalisation noch durch andere hier erfasste Kontrollparameter näher eingrenzbar war. Die Anwendung des Narbenpflasters gestaltete sich problemlos, das Fixiermaterial wurde nicht von allen Patienten als notwendig erachtet. Die Verträglichkeit des Narbenpads selbst war uneingeschränkt gut, bei dem Fixiermaterial ergaben sich bei einer kleinen Zahl von Patienten milde Probleme in Form von Juckreiz und Rötung, die aber nach Absetzen sofort nach 2-3 Tagen wieder rückläufig waren.

Interessante Ergebisse lieferte auch die Auswertung und Zeitdauer der KN. Bei einer groben Unterteilung in „frische keloidale Narben“ (Alter 3-12 Monate) und „alte keloidale Narben“ (> 12 Monate) zeigte sich kein wesentlicher Unterschied. Auch solche $\mathrm{KN}$, die bereits länger als ein Jahr (max. 2 Jahre) bestanden hatten, sprachen in gleicher Weise wie die wenige Monate alten Keloide mit der Verbesserung der aufgeführten Parameter an.

Vermerkt werden muss hier allerdings, dass Aktivitätsparameter wie Rötung, Spannungsgefühl und Juckreiz auch bei diesen alten keloidalen Narben im Sinne der Einschlusskriterien dieser Studie noch vorhanden waren, weshalb verständlicherweise die Patienten auch eine aktive Behandlung wünschten.

Die relativ hohe Akzeptanz des hier geprüften Narbenpads beruht in erster Linie auf der Verbesserung der subjektiv störenden Parameter Juckreiz, Rötung etc. Zwar konnte in einigen Fällen (siehe Abb.1 und 2) eine erhebliche Reduktion der Dicke bis hin zur völligen Abflachung der keloidalen Narben erreicht werden, dennoch waren die Effekte auf das wulstartige Aussehen der keloidalen Narben weniger ausgeprägt, so dass am Ende der Behandlung die eigentliche Narbenwucherung noch erkennbar blieb, wenn sie auch im Volumen deutlich vermindert wurde.

Da die Pathogenese der keloidalen Narben bis heute weitgehend unklar ist [3], kann auch über den detaillierten Ablauf eines mög- 


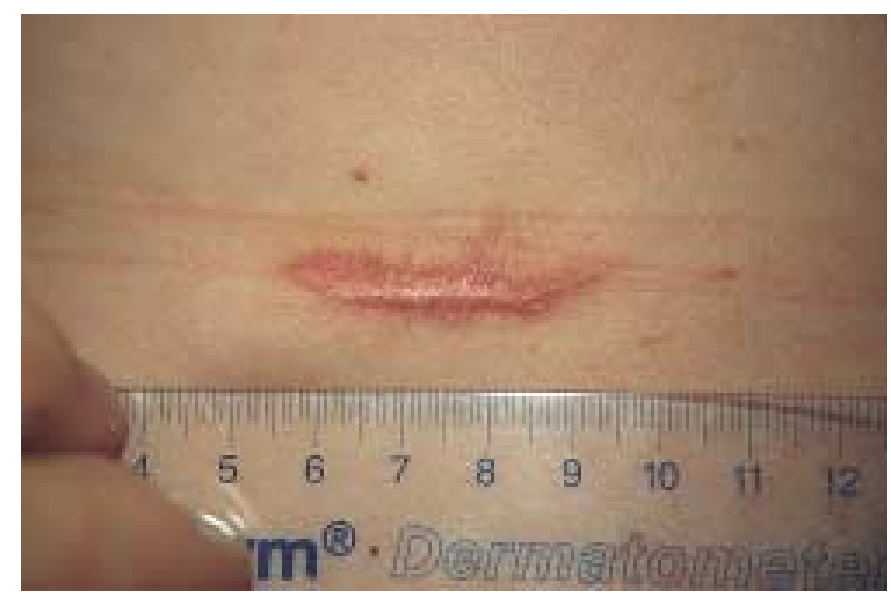

Abb. 3 61-jähriger Mann, im Bauchbereich eine ca. 5 cm lange, mehr als 1 Jahr alte keloidale Narbe.

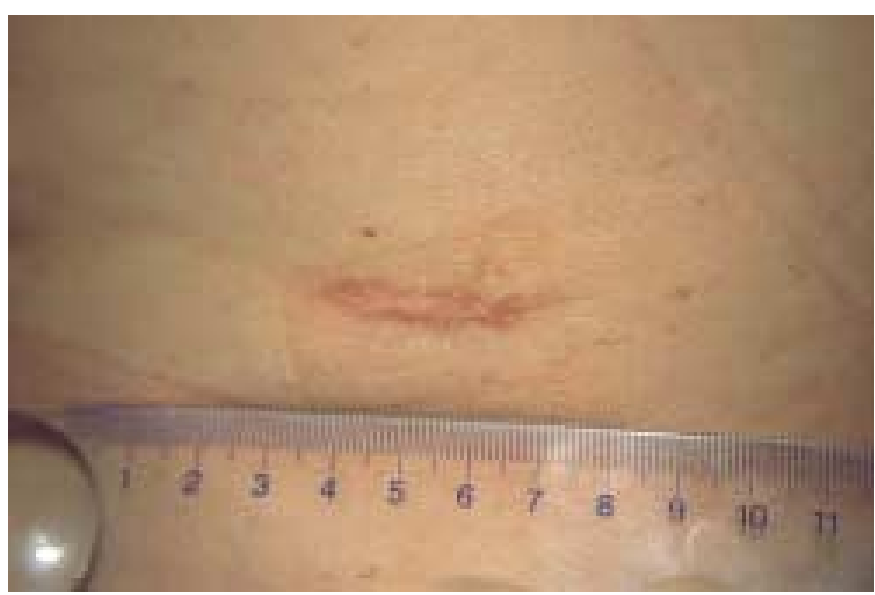

Abb. 4 Ergebnis nach 8 Wochen Behandlung, deutliche Abflachung, vor allen Dingen aber Rückgang der Entzündungszeichen und damit Reduktion der subjektiven Beschwerden vornehmlich des Juckreizes.

lichen Wirkungsmechanismus dieses Narbenpads nur spekuliert werden. Ob die eingangs genannten, genetischen Faktoren in erster Linie die Kollagenproduktion überstimulieren oder die Kollagenolyse am Ende der Narbenbildung (Remodelling) negativ beeinflussen, ist unbekannt. Diverse Untersuchungen konnten aufzeigen, dass sowohl eine gesteigerte Kollagenneosynthese als auch verminderte Kollagenaseaktivität in keloidalen Narben messbar sind, so dass anzunehmenderweise hier eine sich potenzierende gegenseitige Beeinflussung beider Faktoren zum Tragen kommt, an der Integrine, Zytokine und Wachstumsfaktoren (TGF $\beta$ ) und intrazelluläre Matrixkomponenten teilnehmen. $[4,9,16,19]$

Die durch die Okklusion hervorgerufenen Effekte in keloidalen Narben $[14,15]$ scheinen sich vor allem in einer gesteigerten Mikrozirkulation niederzuschlagen. Temperaturmessungen der keloidalen Narben und der Umgebung führten unter Okklusion zu einer Angleichung der Temperatur in den Narben an die der Narbenumgebung [13]. Analog hierzu konnte eine Steigerung der Mikrozirkulation, insbesondere mittels der Venolenflussrate,

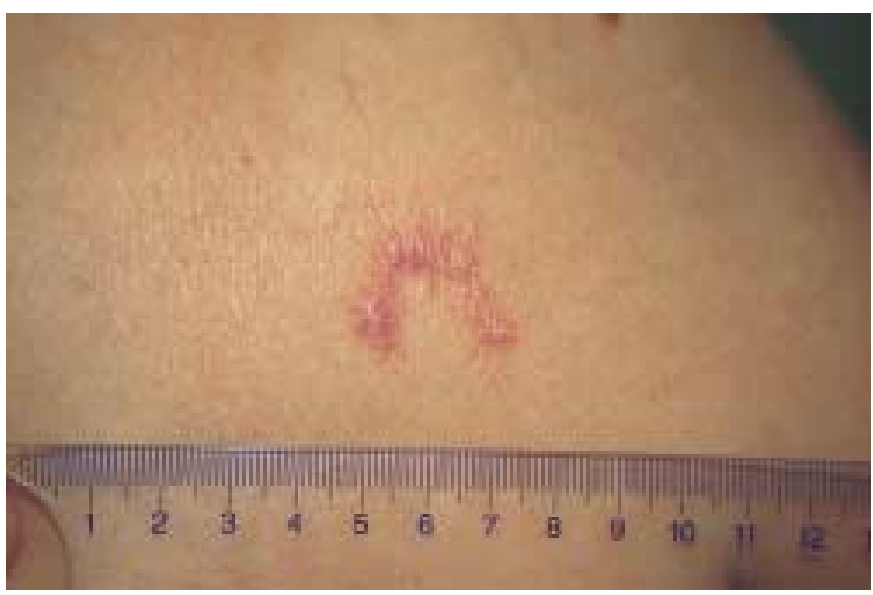

Abb. 5 55-jährige Frau, Zustand nach OP Verschiebelappenplastik 4 Monate zuvor.

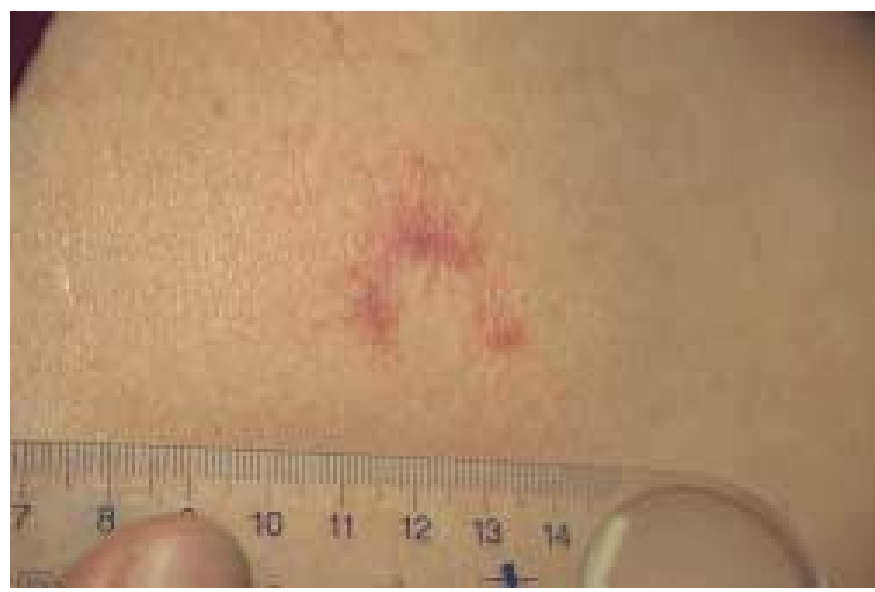

Abb. 6 Nach 10-wöchiger Behandlung Abflachung der Läsion bis ins Hautniveau, noch Reströtungen, weitgehender Rückgang der subjektiven Beschwerden insbesondere Juckreiz. nachgewiesen werden. Im Vergleich zur reinen Kompression waren diese Effekte sowohl bei silikonhaltigen Pflastern als auch bei polyurethanhaltigen Pflastern deutlich nachweisbar, wobei letztere aber noch signifikant besser abschnitten. [1]

Eine Aussage zur grundsätzlichen Beeinflussung der Kollagenneosynthese bzw. strukturellen Veränderung der keloidalen Narben lässt sich aufgrund der vorliegenden Ergebnisse der 8- bis 12-wöchigen Behandlung mit dem Narbenpflaster nicht geben. Frühere Untersuchungen haben gezeigt, dass für die Verbesserung der klinischen Parameter in erster Linie wohl das okklusive Milieu und die damit verbundene Hydratisierung des Gewebes entscheidend ist. Inwieweit hierfür ein Silikonanteil - wie in anderen Präparaten vorhanden - überhaupt erforderlich ist, muss hinterfragt werden, da entsprechende Untersuchungen hierzu keinen wesentlichen Unterschied zwischen silikonfreien und silikonhaltigen Narbenpads aufzeigen konnten. [2]

Die Zukunft der Behandlung keloidaler Narben wird zur Zeit wohl am ehesten in einer Kombination der zur Verfügung ste- 
henden therapeutischen Optionen zu suchen sein [18]. Bei Patienten mit bereits bestehenden Läsionen wird man bei neu anstehenden operativen Eingriffen versuchen, frühzeitig direkt nach dieser OP mit der Behandlung z. B. mit dem hier vorgestellten Narbenpad beginnen, da sich gezeigt hat, dass schon hierdurch ein besseres Narbenbild erreicht werden kann (eigene unveröffentliche Ergebnisse). Zusätzlich wären Unterspritzungen mit Kortikoidkristallsuspensionen nach Ziehen der Fäden nach ca. 10-14 Tage denkbar, um die Proliferation der Fibroblasten zu unterdrücken. Bei beginnender Wulstbildung meist nach 3-4 Wochen könnte eine zusätzliche Therapie mit einem farbstoffgepulsten oder langgepulsten Neodyn:YAK-Laser versucht werden, um die gefäßproliferative Komponente zu reduzieren. Bei bereits bestehenden Keloiden sind die in der Einleitung aufgezählten Methoden allein meist nicht Erfolg bringend. Auch hier wird man Kombinationen aus konservativen (Narbenpflastern) und Kryo-Laser-chirurgische Methoden einsetzen, um die ansonsten eher enttäuschenden Erfolgsquoten steigern zu können.

\section{Literatur}

${ }^{1}$ Baum TM, Busuito MJ. Use of a glycerin-based gel sheeting in Scar Management. Adv in wound care 1998; 11: 40-43

2 Bieley HC, Berman B. Effects of waterimpermeable, non-silicone-based occlusive dressing on keloids. J Am Acad Dermatol 1996; 35: $113-114$

${ }^{3}$ Bock 0, Mrowietz U. Keloide - eine dermale fibroproliferative Erkrankung unbekannter Ursache. Hautarzt 2002; 53: 515-523

${ }^{4}$ Eckes B, Zigrino P, Kessler D, Holtkötter O, Shephard P, Mauch C, Krieg T. Fibroblasmatrix interactions in wound healing and fibrosis. Matrix Biol 2000; 19: 325-332

${ }^{5}$ Ehrlich HP, Desmouliere A, Diegelmann RF, Cohen IK, Compton CC, Garber WL. Morphological and immunochemical differences between keloid and hypertrophic scars. Am J Pathol 1994; 145: 105-113
${ }^{6}$ Espana A, Solano T, Quintanilla E. Bleomycin in the treatment of keloids and hypertrophic scars by multiple needle punctures. Dermatol Surg 2001; 27: 23-27

${ }^{7}$ Farrior RT, Stambaugh KI. Keloids and hyperplastic scars. In: Thomas JR., Holt GR. (Hrsg). Facial scars, revisions, and camouflage. St Louis: CV Mosby, 1989: $211-228$

${ }^{8}$ Fitzpatrick RE. Treatment of inflamed hypertrophic scars using intralesional 5-FU. Dermatol Surg 1999; 25: 224-232

${ }^{9}$ Friedmann DW, Boyd CD, Mackenzie JW, Norton P, Olson RM, Deak SB. Regulation of collagen gene expression in keloids and hypertrophic scars. J Surg Res 1993; 55: 214-222

${ }^{10} \mathrm{Gold} \mathrm{MH}$. A controlled clinical trial of topical silicone gel sheeting in the treatment of hypertrophic scars and keloids. J Am Acad Dermatol 1994; 30: 506-507

${ }^{11}$ Goslen JB. The role of steroids in preventing scar formation. In: Thomas JR., Holt GR (Hrsg). Facial scars, revisions, and camouflage. St. Louis: CV Mosby, 1989: 83-97

${ }^{12}$ Hellwig S. , Raulin C. Gepulste Laser in der Narbenbehandlung. Hautarzt 1999; 50: $465-469$

${ }^{13}$ Klopp R, Niemer W, von der Werth A. Randomized comparative study of the effects of different dressing administrations on the formation of scars after surgical incisions. Wound Repair and Regeneration 1998; 6/5: A473

14 Philipps TJ, Gerstein AD, Lordan V. A randomized controlled trial of hydrocolloid dressing in the treatment of hypertrophic scars and keloids. Dermatol Surg 1996; 22: 775-778

${ }^{15}$ Ricketts CH, Martin L, Faria DT, Saed GM, Fivenson DP. Cytokine mRNA chages during the treatment of hypertrophic scars with silicone and nonsilicone gel dressings. Dermatol Surg 1996; 22: 955 - 959

${ }^{16}$ Roberts AB. Molecular and cell biology of TGF-beta. Miner Electrolyte Metab 1998; 24: 111 - 119

${ }^{17}$ Rusciani L, Rossi G, Bono R. Use of cryotherapy in the treatment of keloids. J Dermatol Surg Oncol 1993; 19: 529-534

${ }^{18}$ Sherris DA, Larrabee WF, Murakami CS. Management of scar contractures, hypertrophic scars, and keloids. Otolaryngol Clin North Am 1995; 28: $1957-1968$

${ }^{19}$ Singer AJ, Clark RAF. Cutaneous wound healing. NEJM 1999; 341: $738-746$

20 Tredget EE, Nedelec B, Scott PG, Ghahary A. Hypertrophic scars, keloids, and contractures. The cellular and molecular basis for therapy. Surg Clin North Am 1997; 77: 701 - 730 